\section{MS15 P01}

Study of Bifurcated Hydrogen Bonding in Co-crystals with Chloranilic acid M. Adam, A. Parkin, \& C.C. Wilson, Dept. of Chemistry \& WestCHEM Research School, University of Glasgow.

E-mail: maadam@chem.gla.ac.uk

Keywords: Hydrogen-bonded molecular adducts, Visualization techniques, X-ray crystallography of samll molecules

X-ray single crystal diffraction is commonly used in structural chemistry. Unfortunately when used conventionally the data obtained lacks the sensitivity to determine accurate hydrogen atom parameters, which is of particular importance in hydrogen-bonded systems. By application of a multi-temperature approach, difference Fourier maps and programs like CrystalExplorer it is possible to gain insight into the temperature-dependent behavior of the hydrogen atoms and their interactions. Indication of the presence of proton disorder and migration can be observed $[1,2]$, determining the best candidate molecules for further study. These are then subjected, where possible, to neutron diffraction analysis to define fully the hydrogen atom behaviour [2]. A structural study of a series of co-crystals with chloranilic acid will be presented. The effects of temperature and $\mathrm{pKa}$ on the bifurcated hydrogen bonds will be discussed and the structures compared using Fourier difference maps, Hirshfeld surfaces and fingerprint plots [3].

[1] C C Wilson \& A E Goeta (2004). Angew Chem Int Ed Engl, 43, 2095-2099; C L Nygren, C C Wilson \& J F C Turner (2005). J Phys Chem A, 109, 2586-2593

[2] A Parkin, S M Harte, A E Goeta \& C C Wilson (2004). New J Chem, 28, 718-721

[3] M.A. Spackman and J.J. McKinnon (2002) CrystEngComm, $4,378-392$

\section{MS15 P02}

Cocrystals of 1,2-Bis(chloromercurio)

Tetrafluorobenzene with Netural Bidentate Lewis Bases. Mikhail Yu. Antipin a,b, Andrey A. Yakovenko ${ }^{\mathrm{a}}$, Jose H. Gallegos ${ }^{\mathrm{a}}$, Tatiana V. Timofeeva ${ }^{\mathrm{a}} \cdot{ }^{a}$ Department of Natural Sciences, New Mexico Highlands University, Las Vegas, NM 87701, USA; ${ }^{b}$ Institute of Organoelement Compounds, Russia Acedemy of Sciences, Moscow, 119991, Russia

E-mail:m_antipin@yahoo.com

In search for extraction agents for Lewis basis we studied cocrystallizastion of Lewis acids and basis. It was found before that the bidentate Lewis acid, 1,2bis(chloromercurio) tetrafluorobenzene $p-\left(\mathrm{C}_{6} \mathrm{~F}_{4}\right)(\mathrm{HgCl})_{2}$ (1) forms complexes with monodentane Lewis bases such as dimetylsulfoxide (DMSO), dimethylformamide (DMF), acetone, dimethyl methylphosphonate (DMMP), benzaldehyde, acetonitrile, THF and propylene oxide. Goal of this project was to find crystallization conditions and structural chartacteristics for complexes of 1 with bidentate Lewis bases including complexes with pharmaceuticals. For example, complex with nitrobenzene (2) $\left\{\left[p-\left(\mathrm{C}_{6} \mathrm{~F}_{4}\right)(\mathrm{HgCl})_{2}\right]\left(\mathrm{PhNO}_{2}\right)\right\}_{\mathrm{n}} \quad$ (3) contains one molecule of a

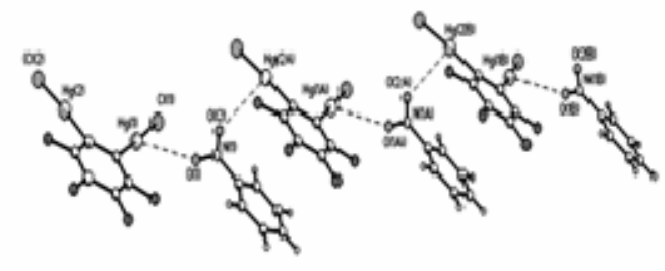

Lewis base per one molecule of $\mathbf{1}$. Complex $\mathbf{3}$ have chiral supramolecular structure (space group $\left(P 2_{1}\right)$, were each oxygen atom of nitro group of $\mathbf{2}$ is coordinated with one mercury atom of $\mathbf{1}$.

\section{MS15 P03 \\ Preparation and Reactivity of Nanosized Cocrystals Formed by Sonocrystallization Dejan-Krešimir Bučar ${ }^{\mathrm{a}}$, Leonard R. MacGillivray, ${ }^{\mathrm{a} D e p a r t m e n t}$ of Chemistry, University of Iowa, Iowa City, IA 52242, USA E-mail: dejan-kresimir-bucar@uiowa.edu}

Keywords: nanocrystalline materials, sonochemistry, photodimerization

Single crystals of macroscopic dimensions are not always appropriate for single-crystal-to-single-crystal (SCSC) reactions due to their mechanical properties (i.e. they crack). A recent study by Nakanishi [1] has described a method to achieve single-crystal-to-single-crystal (SCSC) reactivity within organic crystalline solids by reducing the crystal size to nanodimensions using a precipitation method. Having considered this study, we anticipated that a SCSC [2+2] photoreaction of supramolecular hydrogenbonded assemblies within co-crystals could be achieved by reducing the crystal size to nano- and submicron dimensions. In this contribution, we demonstrate the application of low-intensity ultrasonic radiation to cocrystals of composition 2(resorcinol) 2 (4,4'-bpe) $\mathbf{1}$ [where 4,4'-bpe = trans-1,2-bis(4-pyridyl)ethylene)] through sonocrystallization produces nano- and submicron-sized cocrystals that are shown to exhibit SCSC reactivity [2]. We also demonstrate that that the sonochemical treatment succeeds where sole precipitation fails. The SCSC reactivity of these assemblies was studied by proton nuclear magnetic resonance $\left({ }^{1} \mathrm{H}-\mathrm{NMR}\right)$ and $\mathrm{X}$ ray diffraction (XRPD) while the morphology of the crystalline samples was observed by scanning electron microscopy (SEM).
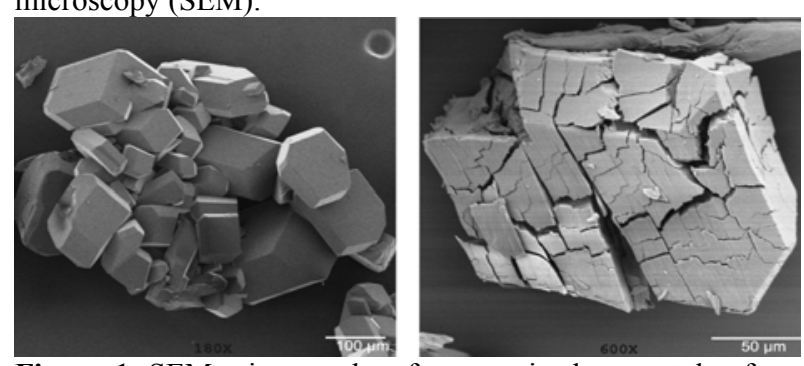

Figure 1. SEM micrographs of macro-sized cocrystals of 1 showing the effects of UV light: (a) before and (b) after photoreaction. 